\title{
Multisynchronization for Coupled Multistable Fractional-Order Neural Networks via Impulsive Control
}

\author{
Jin-E Zhang \\ Hubei Normal University, Hubei 435002, China \\ Correspondence should be addressed to Jin-E Zhang; zhang86021205@163.com
}

Received 1 April 2017; Accepted 5 July 2017; Published 7 August 2017

Academic Editor: Guang Li

Copyright (c) 2017 Jin-E Zhang. This is an open access article distributed under the Creative Commons Attribution License, which permits unrestricted use, distribution, and reproduction in any medium, provided the original work is properly cited.

\begin{abstract}
We show that every subnetwork of a class of coupled fractional-order neural networks consisting of $N$ identical subnetworks can have $(r+1)^{n}$ locally Mittag-Leffler stable equilibria. In addition, we give some algebraic criteria for ascertaining the static multisynchronization of coupled fractional-order neural networks with fixed and switching topologies, respectively. The obtained theoretical results characterize multisynchronization feature for multistable control systems. Two numerical examples are given to verify the superiority of the proposed results.
\end{abstract}

\section{Introduction}

Fractional calculus has been drawing interest over the last decade due to its many applications [1-12]. In mathematics and theoretical physics, fractional calculus is a generalization of integer-order calculus that roots in classical analysis theory. For system modeling, logical mechanism of fractional calculus is more sufficient and more essential $[7,12]$. Then the models involving fractional calculus have greater ability to describe some real phenomena. To go in with mathematical tool behind fractional calculus, we have more room to develop new-type fractional dynamical systems. Actually, long and short memory with power law in dynamic evolution process are usually governed by the socalled fractional dynamical systems [5-7]. Many studies have shown that fractional dynamical systems have an unlimited memory. On the contrary, the memory in integer-order dynamical systems is limited. Moreover, in the general case, the evolution characteristics of fractional dynamical systems cannot be deduced by conventional integer-order variation. Besides, some existing formulations of fractional variation also meets bottleneck: (1) unitarity of evolution operator and (2) temporal fractality. Developing theoretical framework for a breakthrough in analysis and synthesis of fractional dynamical systems is even more important.
Synchronization phenomenon within complex networks is one of the most intriguing and valuable issues [13-16]. Recently, as a special case of an online learning situation, synchronization of neurodynamic systems have been found to be useful in characterizing and validating the consistency of cooperation $[1,8,9,11,12]$. In addition, neural synchronization under setting control rules offers an appealing alternative to neuron architectures and information exchange mechanism during neural mutual learning [16]. However, dynamics of neural synchronization process are intricate. The rhythms of biological brain emerge via synchronization between individual firings by activity-dependent coupling. To get a broader understanding of neural synchronization, it is reasonable to take a closer look at the dynamics of synchronous activity. When neurodynamic systems possess multiple locally stable equilibria, what structure should we use to describe the synchronization manifolds? Moreover, for multistable neurodynamic systems, how to implement this new synchronization control? As far as we know, there is little study. In addition, Lyapunov method $[1,8]$, Razumikhintype stability theory $[9,16]$, infinitesimal generator on analytic semigroup principle [12], adaptive control [17], and mathematical induction method [18] are not very good at estimating the multisynchronization effect. Therefore, for the 
multisynchronization of control systems, there would still be a lot of room left.

It is found that impulsive control is very effective in a wide variety of applications for performance improvement of control process [17-31]. Ayati and Khaloozadeh [19] study the adaptive impulsive control to design an observer for nonlinear continuous systems. Chen et al. [20] address the delayed impulsive control for exponential stability of TakagiSugeno fuzzy systems. Chen et al. [21] show that complex networks can synchronize under the impulsive control policy. It is also demonstrated that second-order consensus can be achieved via impulsive control algorithms [23]. In [24], impulsive control scheme is utilized to improve the performance of differential evolution. In [26], by using the impulsive control method, the ultimate boundedness problem of nonautonomous complex networks is investigated. $\mathrm{Li}$ and Song [27] take full advantage of delay-dependent impulsive control to analyze the stabilization problem of time-delay systems. Liu and Zhang [29] establish the impulsive control principle for stabilization of discrete-time nonlinear systems. Together with comparison criterion, fuzzy impulsive control is used for stabilization of chaotic systems [30]. Impulsive control algorithms are designed for droop-based secondary distributed control in islanded microgrids [31]. In [17], by the impulsive control schemes, exponential synchronization of complex dynamical networks in the presence of stochastic perturbations is formulated. In [18], under delayed impulsive control, stochastic synchronization problem for complex dynamical networks is addressed. Nevertheless, it should be pointed out that the impulsive control for multistable complex systems is still in early stage.

In view of the above discussions, the main objective of this paper is to investigate the multisynchronization for coupled multistable fractional-order neural networks via impulsive control. Several sufficient conditions are obtained to ensure that every subnetwork of coupled fractional-order neural networks has $(2 r+1)^{n}$ equilibria and $(r+1)^{n}$ equilibria are locally Mittag-Leffler stable. The initial-value-related static multisynchronization is introduced to characterize the synchronous behaviors of the controlled coupled multistable fractional-order neural networks. The main emphasis will be then on impulsive control strategy to guarantee multisynchronization for coupled multistable fractional-order neural networks with fixed/switching topology. It is also shown that multisynchronization manifolds can sustain and maintain high levels for long running process. The proposed impulsive control strategy has a number of benefits: (1) saving communication bandwidth; (2) reducing communication cost; (3) good execution performance.

The rest of this paper is organized as follows. In Section 2, preliminaries and problem formulation are given. In Section 3, main results are derived to ascertain multisynchronization for coupled multistable fractional-order neural networks with fixed/switching topology. In Section 4, two numerical examples are presented to show the effectiveness of the obtained results. In Section 5, concluding remarks are stated.

\section{Preliminaries and Problem Formulation}

2.1. Notations. Throughout this paper, ${ }^{C} D_{t_{0}}^{q}(\cdot)$ denotes Caputo fractional derivative operator. $\mathbf{1}^{N}$ is the $N$-dimensional column vector with its elements equal to $1 . I_{k}$ is the $k$ dimensional identity matrix. $\mathscr{A}_{1} \otimes \mathscr{A}_{2}$ denotes the Kronecker product of matrices $\mathscr{A}_{1}$ and $\mathscr{A}_{2}$. Matrix $\mathscr{A}<0(>0 ; \leq 0 ; \geq 0)$ denotes that $\mathscr{A}$ is negative definite (positive definite, negative semidefinite, and positive semidefinite). $\mathscr{A}^{-1}$ represents the inverse matrix of $\mathscr{A}$. For the vector norm or the matrix norm, $\|\cdot\|$ stands for the Euclidean norm.

2.2. Model. Consider a class of coupled fractional-order neural networks consisting of $N$ identical subnetworks

$$
\begin{aligned}
{ }^{C} D_{t_{0}}^{q} x_{i}(t)=-D x_{i}(t)+B F\left(x_{i}(t)\right)+u_{i}(t), & \\
t & \geq t_{0} \geq 0, i=1,2, \ldots, N,
\end{aligned}
$$

where fractional-order $0<q<1, x_{i}(t) \in \mathfrak{R}^{n}$ is the state, $D=\operatorname{diag}\left(d_{1}, d_{2}, \ldots, d_{n}\right)$ is self-feedback term with $d_{k}>$ $0, k=1,2, \ldots, n, B=\left(b_{k l}\right)_{n \times n}$ represents synaptic strength, $F\left(x_{i}(t)\right)=\left(f_{1}\left(x_{i 1}(t)\right), f_{2}\left(x_{i 2}(t)\right), \ldots, f_{n}\left(x_{i n}(t)\right)\right)^{T}$ is the feedback function, and $u_{i}(t) \in \mathfrak{R}^{n}$ denotes the input. The initial value of (1) is given by $x_{i}\left(t_{0}\right)=\left(x_{i 1}\left(t_{0}\right), x_{i 2}\left(t_{0}\right), \ldots, x_{i n}\left(t_{0}\right)\right)^{T}$.

Now, we start to make the following assumptions for (1).

(A1) The feedback functions $F\left(x_{i}(t)\right)=\left(f_{1}\left(x_{i 1}(t)\right)\right.$, $\left.f_{2}\left(x_{i 2}(t)\right), \ldots, f_{n}\left(x_{i n}(t)\right)\right)^{T}, i=1,2, \ldots, N$, satisfy

$$
\begin{aligned}
& \mathscr{P}_{k} \leq f_{k}(s) \leq \widetilde{\mathscr{P}}_{k}, \quad \text { for } \forall s \in \mathfrak{R}, \\
& \mathscr{Q}_{k}^{-} \leq \frac{f_{k}\left(s_{1}\right)-f_{k}\left(s_{2}\right)}{s_{1}-s_{2}} \leq \mathbb{Q}_{k}^{+}, \\
& \qquad \text { for } \forall s_{1}, s_{2} \in \mathfrak{R}, s_{1} \neq s_{2},
\end{aligned}
$$

where $\mathscr{P}_{k}, \widetilde{\mathscr{P}}_{k}, \mathbb{Q}_{k}^{-}$, and $\mathbb{Q}_{k}^{+}, k=1,2, \ldots, n$, are constants.

(A2) $D-\widetilde{B}$ is a nonsingular $M$-matrix, where

$$
\widetilde{B}=\left(\widetilde{b}_{k l}\right)_{n \times n}
$$

with

$$
\begin{aligned}
& \widetilde{b}_{k k}=\max \left\{b_{k k} Q_{k}^{-}, b_{k k} Q_{k}^{+}\right\}, \\
& \widetilde{b}_{k l}=\max \left\{\left|b_{k l}\right|\left|Q_{l}^{-}\right|,\left|b_{k l}\right|\left|Q_{l}^{+}\right|\right\}, \quad k \neq l .
\end{aligned}
$$

(A3) To divide $\mathfrak{R}$ into $2 r+1$ intervals (i.e., $\mathfrak{R}=\left(-\infty, q_{j}^{1}\right) \cup$ $\left.\left[q_{j}^{1}, p_{j}^{1}\right] \cup\left(p_{j}^{1}, q_{j}^{2}\right) \cup\left[q_{j}^{2}, p_{j}^{2}\right] \cup \cdots \cup\left[q_{j}^{r}, p_{j}^{r}\right] \cup\left(p_{j}^{r},+\infty\right)\right)$, then

$$
\begin{aligned}
& -d_{j} q_{j}^{\ell}+b_{j j} f_{j}\left(q_{j}^{\ell}\right)+\sum_{k=1, k \neq j}^{n} \min \left\{b_{j k} \mathscr{P}_{k}, b_{j k} \widetilde{\mathscr{P}}_{k}\right\} \\
& \quad+u_{j}(t)>0, \\
& -d_{j} p_{j}^{\ell}+b_{j j} f_{j}\left(p_{j}^{\ell}\right)+\sum_{k=1, k \neq j}^{n} \max \left\{b_{j k} \mathscr{P}_{k}, b_{j k} \widetilde{\mathscr{P}}_{k}\right\} \\
& \quad+u_{j}(t)<0,
\end{aligned}
$$


for $j=1,2, \ldots, n, \ell=1,2, \ldots, r$, where $-\infty \leq q_{j}^{1}<p_{j}^{1}<$ $q_{j}^{2}<p_{j}^{2}<\cdots<q_{j}^{r}<p_{j}^{r} \leq+\infty$.

Under (A1)-(A3), every subnetwork of system (1) is multistable in Mittag-Leffler sense.

Lemma 1. Let (A1)-(A3) hold, and every subnetwork of system (1) has $(2 r+1)^{n}$ equilibria and $(r+1)^{n}$ equilibria are locally Mittag-Leffler stable.

Using standard arguments as Theorem 1 in [2], Lemma 1 can be proved.

2.3. Properties. In this subsection, we give necessary definition and lemmas.

Denote $S_{1}, S_{2}, \ldots, S_{(r+1)^{n}}$ as $(r+1)^{n}$ locally Mittag-Leffler stable equilibria of every subnetwork of system (1).

Definition 2. System (1) is said to achieve static multisynchronization if the following holds:

(1) For any initial value $x\left(t_{0}\right)=\left(x_{1}^{T}\left(t_{0}\right), x_{2}^{T}\left(t_{0}\right), \ldots\right.$, $\left.x_{N}^{T}\left(t_{0}\right)\right)^{T}$ of $(1)$, where $x_{i}\left(t_{0}\right)=\left(x_{i 1}\left(t_{0}\right), x_{i 2}\left(t_{0}\right), \ldots, x_{i n}\left(t_{0}\right)\right)^{T}$, $i=1,2, \ldots, N$, there exists $S_{\ell} \in \mathfrak{R}^{n}$ such that $\lim _{t \rightarrow+\infty} x_{i}(t)=$ $S_{\ell}, i \in\{1,2, \ldots, N\}, \ell \in\left\{1,2, \ldots,(r+1)^{n}\right\}$.

(2) The synchronization manifolds $\mathbf{1}^{N} \otimes S_{\ell}$ and $\mathbf{1}^{N} \otimes S_{\tilde{\ell}}$ starting from different initial values $x\left(t_{0}\right)=$ $\left(x_{1}^{T}\left(t_{0}\right), x_{2}^{T}\left(t_{0}\right), \ldots, x_{N}^{T}\left(t_{0}\right)\right)^{T}$ and $\widehat{x}\left(t_{0}\right)=\left(\widehat{x}_{1}^{T}\left(t_{0}\right), \widehat{x}_{2}^{T}\left(t_{0}\right), \ldots\right.$, $\left.\widehat{x}_{N}^{T}\left(t_{0}\right)\right)^{T}$, respectively, satisfy the following: there exists $\varepsilon>0$ such that

$$
\begin{aligned}
& \forall \tilde{x}(t) \in\left\{x(t): 0<\left\|x(t)-\mathbf{1}^{N} \otimes S_{\ell}\right\|<\varepsilon, x(t)\right. \\
& \left.\quad \in \mathfrak{R}^{N n}, t \geq t_{0}\right\},
\end{aligned}
$$

where $\widetilde{x}(t)$ is not a point on $\mathbf{1}^{N} \otimes S_{\ell}$.

Lemma 3. Linear matrix inequality

$$
\left[\begin{array}{ll}
\mathscr{A}_{1} & \mathscr{A}_{2} \\
\mathscr{A}_{2}^{T} & \mathscr{A}_{3}
\end{array}\right]<0
$$

is equivalent to

(1) $\mathscr{A}_{1}<0$ and $\mathscr{A}_{3}-\mathscr{A}_{2}^{T} \mathscr{A}_{1}^{-1} \mathscr{A}_{2}<0$,

or

(2) $\mathscr{A}_{3}<0$ and $\mathscr{A}_{1}-\mathscr{A}_{2} \mathscr{A}_{3}^{-1} \mathscr{A}_{2}^{T}<0$,

where $\mathscr{A}_{1}=\mathscr{A}_{1}^{T}$ and $\mathscr{A}_{3}=\mathscr{A}_{3}^{T}$.

Lemma 4. Let $\mathscr{A}(t)$ be a continuous function on $\left[t_{0},+\infty\right)$; if there exists constant $\kappa$ such that

$$
{ }^{C} D_{t_{0}}^{q} \mathscr{A}(t) \leq \kappa \mathscr{A}(t), \quad t \geq t_{0} \geq 0,
$$

then

$$
\mathscr{A}(t) \leq \mathscr{A}\left(t_{0}\right) E_{q}\left(\kappa\left(t-t_{0}\right)^{q}\right), \quad t \geq t_{0} \geq 0,
$$

where $0<q<1, E_{q}(\cdot)$ is one-parameter Mittag-Leffler function.

\section{Main Results}

When the coupled fractional-order neural networks (1) generate multistable behavior, finding out the related multisynchronization control strategy is a challenging issue. In the following, we introduce impulsive control for multisynchronization in (1).

3.1. Fixed Topology Case. For the coupled fractional-order neural networks (1) with fixed topology, the impulsive control design is given below:

$$
u_{i}(t)=a \sum_{h=1}^{+\infty}\left(\sum_{j=1, j \neq i}^{N} c_{i j}\left[x_{j}(t)-x_{i}(t)\right]\right) \delta\left(t-t_{h}\right),
$$

for $i=1,2, \ldots, N$, where $a>0$ is the coupled gain, $c_{i j}$ denotes the element of the weighted adjacency matrix of digraph $\mathscr{D}$, digraph $\mathscr{D}$ possesses a directed spanning tree, $\delta(\cdot)$ is the Dirac Delta function, and the impulsive time sequence $\left\{t_{h}\right\}_{h=1}^{+\infty}$ satisfies $0<t_{1}<t_{2}<\cdots<t_{h}<\cdots$ and $\lim _{h \rightarrow \infty} t_{h}=+\infty$.

Combining with (1) and (11), we get

$$
\begin{array}{r}
{ }^{C} D_{t_{0}}^{q} x(t)=-\left(I_{N} \otimes D\right) x(t)+\left(I_{N} \otimes B\right) F(x(t)), \\
t \neq t_{h}, \\
\Delta x(t)=-\left(a L \otimes I_{n}\right) x\left(t^{-}\right), \quad t=t_{h}, \\
t \geq t_{0} \geq 0, h=1,2, \ldots,
\end{array}
$$

where $x(t)=\left(x_{1}^{T}(t), x_{2}^{T}(t), \ldots, x_{N}^{T}(t)\right)^{T}, F(x(t))=\left(F^{T}\left(x_{1}(t)\right)\right.$, $\left.F^{T}\left(x_{2}(t)\right), \ldots, F^{T}\left(x_{N}(t)\right)\right)^{T}$, and $L=\left(\mathscr{L}_{i j}\right)_{N \times N}$ is the Laplacian matrix associated with digraph $\mathscr{D}$.

Theorem 5. Let (A1)-(A3) hold; for any given constant $\alpha_{1}>0$, if there exist constant $0<\alpha_{2}<1$ and matrices $\mathscr{R}>0$ and $\mathscr{W}=\operatorname{diag}\left(\mathscr{W}_{1}, \mathscr{W}_{2}, \ldots, \mathscr{W}_{n}\right)>0$ such that

$$
\left[\begin{array}{cc}
-\alpha_{2} I_{N-1} & \left(I_{N-1}-a \mathcal{S}\right)^{T} \\
\left(I_{N-1}-a \mathcal{S}\right) & -I_{N-1}
\end{array}\right]<0
$$

$$
\left[\begin{array}{cc}
-\mathscr{R} D-D^{T} \mathscr{R}+\frac{\alpha_{2}}{\alpha_{1}} \mathscr{R}-\mathscr{W} \mathscr{M}_{1} & \left(\mathscr{R} B+\mathscr{W} \mathscr{M}_{2}\right) \\
\left(\mathscr{R} B+\mathscr{W} \mathscr{M}_{2}\right)^{T} & -\mathscr{W}
\end{array}\right]
$$

$<0$,

where

$$
\begin{aligned}
& \mathcal{S}=\left(\mathscr{L}_{i j}-\mathscr{L}_{N j}\right)_{(N-1) \times(N-1)}, \\
& \mathscr{M}_{1}=\operatorname{diag}\left(\widehat{Q}_{1}^{+} Q_{1}^{-}, Q_{2}^{+} Q_{2}^{-}, \ldots, Q_{n}^{+} Q_{n}^{-}\right), \\
& \mathscr{M}_{2}=\operatorname{diag}\left(\frac{\mathscr{Q}_{1}^{+}+{Q_{1}^{-}}_{1}}{2}, \frac{\mathscr{Q}_{2}^{+}+{Q_{2}^{-}}_{2}}{2}, \ldots, \frac{\widehat{Q}_{n}^{+}+\mathscr{Q}_{n}^{-}}{2}\right) \text {, }
\end{aligned}
$$

then, for the impulsive time sequence $\left\{t_{h}\right\}_{h=1}^{+\infty}$ admitting $\sup \left\{t_{h}-\right.$ $\left.t_{h-1}\right\} \leq \alpha_{1}$, system (12) can achieve static multisynchronization. 
Proof. From (A1)-(A3), according to Lemma 1, every subnetwork of system (1) has $(r+1)^{n}$ locally Mittag-Leffler stable equilibria $S_{1}, S_{2}, \ldots, S_{(r+1)^{n}}$.

Let $\mathscr{Y}_{i}(t)=x_{i}(t)-S, i=1,2, \ldots, N$, where $S \in\left\{S_{\hbar}, \hbar=\right.$ $\left.1,2, \ldots,(r+1)^{n}\right\}$, from (12), and then

$$
\begin{array}{r}
{ }^{C} D_{t_{0}}^{q} \mathscr{Y}(t)=-\left(I_{N} \otimes D\right) \mathscr{Y}(t)+\left(I_{N} \otimes B\right) F(\mathscr{Y}(t)), \\
t \neq t_{h}, \\
\Delta \mathscr{Y}(t)=-\left(a L \otimes I_{n}\right) \mathscr{Y}\left(t^{-}\right), \quad t=t_{h}, \\
t \geq t_{0} \geq 0, h=1,2, \ldots,
\end{array}
$$

where $\mathscr{Y}(t)=\left(\mathscr{Y}_{1}^{T}(t), \mathscr{Y}_{2}^{T}(t), \ldots, \mathscr{Y}_{N}^{T}(t)\right)^{T}$ and $F(y(t))=$ $\left(F^{T}\left(\mathscr{Y}_{1}(t)\right), F^{T}\left(\mathscr{Y}_{2}(t)\right), \ldots, F^{T}\left(\mathscr{Y}_{N}(t)\right)\right)^{T}$.

Let $\mathscr{X}_{i}(t)=\mathscr{Y}_{i}(t)-\mathscr{Y}_{N}(t), i=1,2, \ldots, N-1$, and system (16) can be reformulated as

$$
\begin{aligned}
&{ }^{C} D_{t_{0}}^{q} \mathscr{Z}(t)=-\left(I_{N-1} \otimes D\right) \mathscr{Z}(t) \\
&+\left(I_{N-1} \otimes B\right) F(\mathscr{E}(t)), \quad t \neq t_{h}, \\
&{ }^{C} D_{t_{0}}^{q} \mathscr{Y}_{N}(t)=-D \mathscr{Y}_{N}(t)+B F\left(\mathscr{Y}_{N}(t)\right), \quad t \neq t_{h}, \\
& \Delta \mathscr{Z}(t)=-\left(a \mathcal{S} \otimes I_{n}\right) \mathscr{Z}\left(t^{-}\right), \quad t=t_{h}, \\
& \Delta \mathscr{Y}_{N}(t)=-\left(a \widehat{\mathcal{S}} \otimes I_{n}\right) \mathscr{Y}_{N}\left(t^{-}\right), \quad t=t_{h}, \\
& t \geq t_{0} \geq 0, h=1,2, \ldots,
\end{aligned}
$$

where $\mathscr{E}(t)=\left(\mathscr{E}_{1}^{T}(t), \mathscr{E}_{2}^{T}(t), \ldots, \mathscr{E}_{N-1}^{T}(t)\right)^{T}, F(\mathscr{Z}(t))=$ $\left(F^{T}\left(\mathscr{Z}_{1}(t)\right), F^{T}\left(\mathscr{Z}_{2}(t)\right), \ldots, F^{T}\left(\mathscr{Z}_{N-1}(t)\right)\right)^{T}$, and $\widehat{\mathcal{S}}=\left(\mathscr{L}_{N 1}\right.$, $\left.\mathscr{L}_{N 2}, \ldots, \mathscr{L}_{N(N-1)}\right)$.

Obviously, for $i=1,2, \ldots, N-1$,

$$
\begin{aligned}
\mathscr{Z}_{i}(t) & =\mathscr{Y}_{i}(t)-\mathscr{Y}_{N}(t)=\left(x_{i}(t)-S\right)-\left(x_{N}(t)-S\right) \\
& =x_{i}(t)-x_{N}(t) .
\end{aligned}
$$

According to Lemma 3, (13) is equivalent to

$$
\left(I_{N-1}-a \mathcal{S}\right)^{T}\left(I_{N-1}-a \mathcal{S}\right) \leq \alpha_{2} I_{N-1} .
$$

Define a Lyapunov function

$$
V(t)=\mathscr{Z}^{T}(t)\left(I_{N-1} \otimes \mathscr{R}\right) \mathscr{Z}(t) .
$$

When $t=t_{h}$, we can obtain

$$
\begin{aligned}
V\left(t_{h}\right)= & \mathscr{E}^{T}\left(t_{h}\right)\left(I_{N-1} \otimes \mathscr{R}\right) \mathscr{Z}\left(t_{h}\right) \\
= & \mathscr{E}^{T}\left(t_{h}^{-}\right)\left[\left(I_{N-1}-a \mathcal{S}\right) \otimes I_{h}\right]^{T}\left(I_{N-1} \otimes \mathscr{R}\right) \\
& \times\left[\left(I_{N-1}-a \delta\right) \otimes I_{n}\right] \mathscr{Z}\left(t_{h}^{-}\right) \leq \alpha_{2} V\left(t_{h}^{-}\right) .
\end{aligned}
$$

On the other hand, by (3) in (A1), for any given $\mathscr{W}=$ $\operatorname{diag}\left(\mathscr{W}_{1}, \mathscr{W}_{2}, \ldots, \mathscr{W}_{n}\right)>0$, we have

$$
\begin{aligned}
0 \leq & \sum_{j=1}^{n} \mathscr{W}_{j}\left[\mathscr{Q}_{j}^{+} \mathscr{Z}_{j}(t)-F\left(\mathscr{Z}_{j}(t)\right)\right] \\
& \cdot\left[F\left(\mathscr{Z}_{j}(t)\right)-\mathscr{Q}_{j}^{-} \mathscr{Z}_{j}(t)\right]=-\mathscr{E}_{i}^{T}(t) \mathscr{W} \mathscr{M}_{1} \mathscr{Z}_{i}(t) \\
& +2 \mathscr{Z}_{i}^{T}(t) \mathscr{W} \mathscr{M}_{2} F\left(\mathscr{Z}_{i}(t)\right)-F^{T}\left(\mathscr{Z}_{i}(t)\right) \\
& \cdot \mathscr{W} F\left(\mathscr{Z}_{i}(t)\right),
\end{aligned}
$$

and thus

$$
\begin{aligned}
0 \leq & -\mathscr{Z}^{T}(t) I_{N-1} \otimes\left(\mathscr{W} \mathscr{M}_{1}\right) \mathscr{Z}(t)+2 \mathscr{Z}^{T}(t) I_{N-1} \\
& \otimes\left(\mathscr{W} \mathscr{M}_{2}\right) F(\mathscr{E}(t))-F^{T}(\mathscr{E}(t)) I_{N-1} \\
& \otimes \mathscr{W} F(\mathscr{E}(t)) .
\end{aligned}
$$

By (14), it follows that

$$
\left[\begin{array}{cc}
-\mathscr{R} D-D^{T} \mathscr{R}+\frac{\left(\alpha_{2}+\alpha_{3}\right)}{\alpha_{1}} \mathscr{R}-\mathscr{W} \mathscr{M}_{1} & \left(\mathscr{R} B+\mathscr{W} \mathscr{M}_{2}\right) \\
\left(\mathscr{R} B+\mathscr{W} \mathscr{M}_{2}\right)^{T} & -\mathscr{W}
\end{array}\right]
$$

$<0$,

where constant $\alpha_{3} \in\left(0,1-\alpha_{2}\right)$.

When $t \neq t_{h}$, we can get

$$
\begin{aligned}
{ }^{C} D_{t_{0}}^{q} V(t) \leq 2 \mathscr{Z}^{T}(t)\left(I_{N-1} \otimes \mathscr{R}\right) \mathscr{Z}(t)+2 \mathscr{Z}^{T}(t) \\
\cdot\left(I_{N-1} \otimes \mathscr{R}\right) \\
\cdot\left[-\left(I_{N-1} \otimes D\right) \mathscr{Z}(t)+\left(I_{N-1} \otimes B\right) F(\mathscr{Z}(t))\right] .
\end{aligned}
$$

Together with (23)-(25),

$$
\begin{aligned}
{ }^{C} D_{t_{0}}^{q} V(t) \leq & \mathscr{H}^{T}(t)\left(I_{N-1} \otimes \chi\right) \mathscr{H}(t) \\
& -\frac{\left(\alpha_{2}+\alpha_{3}\right)}{\alpha_{1}} V(t)<-\frac{\left(\alpha_{2}+\alpha_{3}\right)}{\alpha_{1}} V(t),
\end{aligned}
$$

where

$$
\begin{aligned}
& \mathscr{H}^{T}(t)=\left(\mathscr{Z}^{T}(t), F^{T}(\mathscr{X}(t))\right)^{T}, \\
& \chi \\
& =\left[\begin{array}{cc}
-\mathscr{R} D-D^{T} \mathscr{R}+\frac{\left(\alpha_{2}+\alpha_{3}\right)}{\alpha_{1}} \mathscr{R}-\mathscr{W} \mathscr{M}_{1}\left(\mathscr{R} B+\mathscr{W} \mathscr{M}_{2}\right) \\
\left(\mathscr{R} B+\mathscr{W} \mathscr{M}_{2}\right)^{T} & -\mathscr{W}
\end{array}\right] .
\end{aligned}
$$

According to Lemma 4,

$V(t) \leq V\left(t_{0}\right) E_{q}\left(-\frac{\left(\alpha_{2}+\alpha_{3}\right)}{\alpha_{1}}\left(t-t_{0}\right)^{q}\right)$,

$$
t \geq t_{0} \geq 0,
$$

so $V(t) \rightarrow 0$ as $t \rightarrow+\infty$, which implies that, for any given initial value of (1), $x_{1}(t)=x_{2}(t)=\cdots=x_{N}(t)$ as $t \rightarrow+\infty$, and, hence, system (12) can reach complete synchronization. 
Moreover, consider the Nth subnetwork of (12):

$$
\begin{aligned}
{ }^{C} D_{t_{0}}^{q} x_{N}(t) & =-D x_{N}(t)+B F\left(x_{N}(t)\right), \quad t \neq t_{h}, \\
\Delta x_{N}(t) & =\sum_{j=1}^{N-1} c_{N j}\left(x_{j}\left(t^{-}\right)-x_{N}\left(t^{-}\right)\right), \quad t=t_{h},
\end{aligned}
$$

$$
t \geq t_{0} \geq 0, h=1,2, \ldots
$$

Based on the above analysis, we have $\Delta x_{N}(t)=0$ as $t \rightarrow+\infty$. Through Lemma 1 , the $N$ th subnetwork of (12) has $(r+1)^{n}$ locally Mittag-Leffler stable equilibria $S_{1}, S_{2}$, ldots, $S_{(r+1)^{n}}$. Therefore, it follows that $x_{1}(t)=$ $x_{2}(t)=\cdots=x_{N}(t)=S$ as $t \rightarrow+\infty, S \in\left\{S_{\hbar}, \hbar=\right.$ $\left.1,2, \ldots,(r+1)^{n}\right\}$. To sum up, system (12) can achieve static multisynchronization.

3.2. Switching Topology Case. Without loss of generality, we introduce a switching signal $\rho(t):\left[t_{0},+\infty\right) \rightarrow\{1,2, \ldots, \mathscr{K}\}$ and $\mathscr{K}$ digraphs indexed by digraph $\mathscr{D}^{1}$, digraph $\mathscr{D}^{2}, \ldots$, digraph $\mathscr{D}^{\mathscr{K}}$.

For the coupled fractional-order neural networks (1) with switching topology, the impulsive control design is given below:

$$
\begin{aligned}
& u_{i}(t) \\
& \quad=a^{\rho(t)} \sum_{h=1}^{+\infty}\left(\sum_{j=1, j \neq i}^{N} c_{i j}^{\rho(t)}\left[x_{j}(t)-x_{i}(t)\right]\right) \delta\left(t-t_{h}\right),
\end{aligned}
$$

for $i=1,2, \ldots, N$, where the switching signal $\rho(t)$ $\left[t_{0},+\infty\right) \rightarrow\{1,2, \ldots, \mathscr{K}\}, a^{\rho(t)}>0$ is the coupled gain, $c_{i j}^{\rho(t)}$ denotes the element of the weighted adjacency matrix of digraph $\mathscr{D}^{\rho(t)}$, digraph $\mathscr{D}^{\rho(t)}$ possesses a directed spanning tree, $\delta(\cdot)$ is the Dirac Delta function, and the impulsive time sequence $\left\{t_{h}\right\}_{h=1}^{+\infty}$ satisfies $0<t_{1}<t_{2}<\cdots<t_{h}<\cdots$ and $\lim _{h \rightarrow \infty} t_{h}=+\infty$.

Combining with (1) and (30), we get

$$
\begin{array}{r}
{ }^{C} D_{t_{0}}^{q} x(t)=-\left(I_{N} \otimes D\right) x(t)+\left(I_{N} \otimes B\right) F(x(t)), \\
t \neq t_{h}, \\
\Delta x(t)=-\left(a^{\rho(t)} L^{\rho(t)} \otimes I_{n}\right) x\left(t^{-}\right), \quad t=t_{h}, \\
t \geq t_{0} \geq 0, h=1,2, \ldots,
\end{array}
$$

where $x(t)=\left(x_{1}^{T}(t), x_{2}^{T}(t), \ldots, x_{N}^{T}(t)\right)^{T}, F(x(t))=\left(F^{T}\left(x_{1}(t)\right)\right.$, $\left.F^{T}\left(x_{2}(t)\right), \ldots, F^{T}\left(x_{N}(t)\right)\right)^{T}$, and $L^{\rho(t)}=\left(\mathscr{L}_{i j}^{\rho(t)}\right)_{N \times N}$ is the Laplacian matrix associated with digraph $\mathscr{D}^{\rho(t)}$.
Theorem 6. Let (A1)-(A3) hold; for any given constant $\alpha_{1}>0$, if there exist constant $0<\alpha_{2}<1$ and matrices $\mathscr{R}>0$ and $\mathscr{W}=\operatorname{diag}\left(\mathscr{W}_{1}, \mathscr{W}_{2}, \ldots, \mathscr{W}_{n}\right)>0$ such that

$$
\begin{aligned}
& {\left[\begin{array}{cc}
-\alpha_{2} I_{N-1} & \left(I_{N-1}-a^{\mathscr{C}} \mathcal{S}^{\mathscr{C}}\right)^{T} \\
\left(I_{N-1}-a^{\mathscr{C}} \mathcal{S}^{\mathscr{C}}\right) & -I_{N-1}
\end{array}\right]<0,} \\
& \mathscr{C}=1,2, \ldots, \mathscr{K}, \\
& {\left[\begin{array}{cc}
-\mathscr{R} D-D^{T} \mathscr{R}+\frac{\alpha_{2}}{\alpha_{1}} \mathscr{R}-\mathscr{W} \mathscr{M}_{1} & \left(\mathscr{R} B+\mathscr{W} \mathscr{M}_{2}\right) \\
\left(\mathscr{R} B+\mathscr{W} \mathscr{M}_{2}\right)^{T} & -\mathscr{W}
\end{array}\right]} \\
& <0,
\end{aligned}
$$

where

$$
\begin{aligned}
& \mathcal{S}^{\mathscr{C}}=\left(\mathscr{L}_{i j}^{\mathscr{C}}-\mathscr{L}_{N j}^{\mathscr{C}}\right)_{(N-1) \times(N-1)}, \quad \mathscr{C}=1,2, \ldots, \mathscr{K}, \\
& \mathscr{M}_{1}=\operatorname{diag}\left(Q_{1}^{+} Q_{1}^{-}, Q_{2}^{+} Q_{2}^{-}, \ldots,{Q_{n}^{+}}_{n}^{-}\right), \\
& \mathscr{M}_{2}=\operatorname{diag}\left(\frac{Q_{1}^{+}+Q_{1}^{-}}{2}, \frac{Q_{2}^{+}+Q_{2}^{-}}{2}, \ldots, \frac{\mathscr{Q}_{n}^{+}+Q_{n}^{-}}{2}\right),
\end{aligned}
$$

then, for the impulsive time sequence $\left\{t_{h}\right\}_{h=1}^{+\infty}$ admitting $\sup \left\{t_{h}-\right.$ $\left.t_{h-1}\right\} \leq \alpha_{1}$, system (31) can achieve static multisynchronization.

Proof. From (A1)-(A3), according to Lemma 1, every subnetwork of system (1) has $(r+1)^{n}$ locally Mittag-Leffler stable equilibria $S_{1}, S_{2}, \ldots, S_{(r+1)^{n}}$.

Let $\mathscr{Y}_{i}(t)=x_{i}(t)-S, i=1,2, \ldots, N$, where $S \in\left\{S_{\hbar}, \hbar=\right.$ $\left.1,2, \ldots,(r+1)^{n}\right\}$; from (31), then

$$
\begin{array}{r}
{ }^{C} D_{t_{0}}^{q} \mathcal{Y}(t)=-\left(I_{N} \otimes D\right) \mathcal{Y}(t)+\left(I_{N} \otimes B\right) F(\mathcal{Y}(t)), \\
t \neq t_{h}, \\
\Delta \mathscr{Y}(t)=-\left(a^{\rho(t)} L^{\rho(t)} \otimes I_{n}\right) \mathcal{Y}\left(t^{-}\right), \quad t=t_{h}, \\
t \geq t_{0} \geq 0, h=1,2, \ldots,
\end{array}
$$

where $\mathscr{Y}(t)=\left(\mathcal{Y}_{1}^{T}(t), \mathscr{Y}_{2}^{T}(t), \ldots, \mathscr{Y}_{N}^{T}(t)\right)^{T}$ and $F(\mathcal{Y}(t))=$ $\left(F^{T}\left(\mathscr{Y}_{1}(t)\right), F^{T}\left(\mathscr{Y}_{2}(t)\right), \ldots, F^{T}\left(\mathscr{Y}_{N}(t)\right)\right)^{T}$.

Let $\mathscr{Z}_{i}(t)=\mathscr{Y}_{i}(t)-\mathscr{Y}_{N}(t), i=1,2, \ldots, N-1$, and system (35) can be reformulated as

$$
\begin{aligned}
&{ }^{C} D_{t_{0}}^{q} \mathscr{Z}(t)=-\left(I_{N-1} \otimes D\right) \mathscr{Z}(t) \\
&+\left(I_{N-1} \otimes B\right) F(\mathscr{Z}(t)), \quad t \neq t_{h}, \\
&{ }^{C} D_{t_{0}}^{q} \mathscr{Y}_{N}(t)=-D \mathscr{Y}_{N}(t)+B F\left(\mathscr{Y}_{N}(t)\right), \quad t \neq t_{h}, \\
& \Delta \mathscr{Z}(t)=-\left(a^{\rho(t)} \mathcal{S}^{\rho(t)} \otimes I_{n}\right) \mathscr{Z}\left(t^{-}\right), \quad t=t_{h}, \\
& \Delta \mathscr{Y}_{N}(t)=-\left(a^{\rho(t)} \widehat{\mathcal{S}}^{\rho(t)} \otimes I_{n}\right) \mathscr{Y}_{N}\left(t^{-}\right), \quad t=t_{h}, \\
& t \geq t_{0} \geq 0, h=1,2, \ldots,
\end{aligned}
$$

where $\mathscr{Z}(t)=\left(\mathscr{Z}_{1}^{T}(t), \mathscr{Z}_{2}^{T}(t), \ldots, \mathscr{Z}_{N-1}^{T}(t)\right)^{T}, F(\mathscr{Z}(t))=$ $\left(F^{T}\left(\mathscr{Z}_{1}(t)\right), F^{T}\left(\mathscr{Z}_{2}(t)\right), \ldots, F^{T}\left(\mathscr{Z}_{N-1}(t)\right)\right)^{T}$, and $\widehat{\mathcal{S}}^{\rho(t)}=$ $\left(\mathscr{L}_{N 1}^{\rho(t)}, \mathscr{L}_{N 2}^{\rho(t)}, \ldots, \mathscr{L}_{N(N-1)}^{\rho(t)}\right)$. 
Obviously, for $i=1,2, \ldots, N-1$,

$$
\begin{aligned}
\mathscr{X}_{i}(t) & =\mathscr{Y}_{i}(t)-\mathscr{Y}_{N}(t)=\left(x_{i}(t)-S\right)-\left(x_{N}(t)-S\right) \\
& =x_{i}(t)-x_{N}(t) .
\end{aligned}
$$

According to Lemma 3, (32) is equivalent to

$$
\left(I_{N-1}-a^{\mathscr{C}} \mathcal{S}^{\mathscr{C}}\right)^{T}\left(I_{N-1}-a^{\mathscr{C}} \mathcal{S}^{\mathscr{C}}\right) \leq \alpha_{2} I_{N-1},
$$

$$
\mathscr{C}=1,2, \ldots, \mathscr{K} \text {. }
$$

Define a Lyapunov function

$$
V(t)=\mathscr{Z}^{T}(t)\left(I_{N-1} \otimes \mathscr{R}\right) \mathscr{Z}(t) .
$$

When $t=t_{h}$, we can obtain

$$
\begin{aligned}
V\left(t_{h}\right)= & \mathscr{Z}^{T}\left(t_{h}\right)\left(I_{N-1} \otimes \mathscr{R}\right) \mathscr{Z}\left(t_{h}\right) \\
= & \mathscr{Z}^{T}\left(t_{h}^{-}\right)\left[\left(I_{N-1}-a^{\mathscr{C}} \mathcal{S}^{\mathscr{C}}\right) \otimes I_{n}\right]^{T}\left(I_{N-1} \otimes \mathscr{R}\right) \\
& \times\left[\left(I_{N-1}-a^{\mathscr{C}} \mathcal{S}^{\mathscr{C}}\right) \otimes I_{n}\right] \mathscr{Z}\left(t_{h}^{-}\right) \\
\leq & \alpha_{2} V\left(t_{h}^{-}\right) .
\end{aligned}
$$

On the other hand, by (3) in (A1), for any given $\mathscr{W}=$ $\operatorname{diag}\left(\mathscr{W}_{1}, \mathscr{W}_{2}, \ldots, \mathscr{W}_{n}\right)>0$, we have

$$
\begin{aligned}
0 \leq & \sum_{j=1}^{n} \mathscr{W}_{j}\left[\mathscr{Q}_{j}^{+} \mathscr{Z}_{j}(t)-F\left(\mathscr{Z}_{j}(t)\right)\right] \\
& \cdot\left[F\left(\mathscr{Z}_{j}(t)\right)-\mathscr{Q}_{j}^{-} \mathscr{Z}_{j}(t)\right]=-\mathscr{Z}_{i}^{T}(t) \mathscr{W} \mathscr{M}_{1} \mathscr{Z}_{i}(t) \\
& +2 \mathscr{Z}_{i}^{T}(t) \mathscr{W} \mathscr{M}_{2} F\left(\mathscr{Z}_{i}(t)\right)-F^{T}\left(\mathscr{Z}_{i}(t)\right) \\
& \cdot \mathscr{W} F\left(\mathscr{Z}_{i}(t)\right),
\end{aligned}
$$

and thus

$$
\begin{aligned}
0 \leq & -\mathscr{Z}^{T}(t) I_{N-1} \otimes\left(\mathscr{W} \mathscr{M}_{1}\right) \mathscr{Z}(t)+2 \mathscr{Z}^{T}(t) I_{N-1} \\
& \otimes\left(\mathscr{W} \mathscr{M}_{2}\right) F(\mathscr{Z}(t))-F^{T}(\mathscr{Z}(t)) I_{N-1} \\
& \otimes \mathscr{W} F(\mathscr{Z}(t)) .
\end{aligned}
$$

By (33), it follows that

$$
\left[\begin{array}{cc}
-\mathscr{R} D-D^{T} \mathscr{R}+\frac{\left(\alpha_{2}+\alpha_{3}\right)}{\alpha_{1}} \mathscr{R}-\mathscr{W} \mathscr{M}_{1} & \left(\mathscr{R} B+\mathscr{W} \mathscr{M}_{2}\right) \\
\left(\mathscr{R} B+\mathscr{W} \mathscr{M}_{2}\right)^{T} & -\mathscr{W}
\end{array}\right]
$$

where constant $\alpha_{3} \in\left(0,1-\alpha_{2}\right)$.

When $t \neq t_{h}$, we can get

$$
\begin{aligned}
{ }^{C} D_{t_{0}}^{q} V(t) \leq 2 \mathscr{Z}^{T}(t)\left(I_{N-1} \otimes \mathscr{R}\right) \mathscr{Z}(t)+2 \mathscr{Z}^{T}(t) \\
\cdot\left(I_{N-1} \otimes \mathscr{R}\right) \\
\cdot\left[-\left(I_{N-1} \otimes D\right) \mathscr{Z}(t)+\left(I_{N-1} \otimes B\right) F(\mathscr{Z}(t))\right] .
\end{aligned}
$$

Together with (42)-(44),

$$
\begin{aligned}
{ }^{C} D_{t_{0}}^{q} V(t) \leq & \mathscr{H}^{T}(t)\left(I_{N-1} \otimes \chi\right) \mathscr{H}(t) \\
& -\frac{\left(\alpha_{2}+\alpha_{3}\right)}{\alpha_{1}} V(t)<-\frac{\left(\alpha_{2}+\alpha_{3}\right)}{\alpha_{1}} V(t),
\end{aligned}
$$

where

$$
\mathscr{H}^{T}(t)=\left(\mathscr{Z}^{T}(t), F^{T}(\mathscr{Z}(t))\right)^{T},
$$

$\chi$

$$
=\left[\begin{array}{cc}
-\mathscr{R} D-D^{T} \mathscr{R}+\frac{\left(\alpha_{2}+\alpha_{3}\right)}{\alpha_{1}} \mathscr{R}-\mathscr{W} \mathscr{M}_{1} & \left(\mathscr{R} B+\mathscr{W} \mathscr{M}_{2}\right) \\
\left(\mathscr{R} B+\mathscr{W} \mathscr{M}_{2}\right)^{T} & -\mathscr{W}
\end{array}\right] .
$$

According to Lemma 4,

$$
\begin{aligned}
V(t) \leq V\left(t_{0}\right) E_{q}\left(-\frac{\left(\alpha_{2}+\alpha_{3}\right)}{\alpha_{1}}\left(t-t_{0}\right)^{q}\right), & \\
& t \geq t_{0} \geq 0,
\end{aligned}
$$

so $V(t) \rightarrow 0$ as $t \rightarrow+\infty$, which implies that, for any given initial value of (1), $x_{1}(t)=x_{2}(t)=\cdots=x_{N}(t)$ as $t \rightarrow+\infty$, and, hence, system (31) can reach complete synchronization.

Moreover, consider the Nth subnetwork of (31):

$$
\begin{array}{r}
{ }^{C} D_{t_{0}}^{q} x_{N}(t)=-D x_{N}(t)+B F\left(x_{N}(t)\right), \quad t \neq t_{h}, \\
\Delta x_{N}(t)=\sum_{j=1}^{N-1} c_{N j}^{\rho(t)}\left(x_{j}\left(t^{-}\right)-x_{N}\left(t^{-}\right)\right), \quad t=t_{h}, \\
t \geq t_{0} \geq 0, h=1,2, \ldots .
\end{array}
$$

Based on the above analysis, we have $\Delta x_{N}(t)=0$ as $t \rightarrow+\infty$. Through Lemma 1 , the $N$ th subnetwork of (31) has $(r+1)^{n}$ locally Mittag-Leffler stable equilibria $S_{1}, S_{2}$, $\ldots, S_{(r+1)^{n}}$. Therefore, it follows that $x_{1}(t)=x_{2}(t)=\cdots=$ $x_{N}(t)=S$ as $t \rightarrow+\infty, S \in\left\{S_{\hbar}, \hbar=1,2, \ldots,(r+1)^{n}\right\}$. To sum up, system (31) can achieve static multisynchronization.

Remark 7. Linear matrix inequality has emerged as a very powerful tool and design technique for a lot of the control problems. From the viewpoint of mathematics, the linear matrix inequality is a convex constraint. So the computational procedure scheme for linear matrix inequality can be processed efficiently. A much more effective computing method for solving these kinds of issues is the interior point method [32]. By using Newton's method, the interior point method transforms the constrained optimization problem into an unconstrained optimization problem to be solved. Accordingly, reducing a control design problem to the linear matrix inequality may be a practical approach to this problem [33]. Given that, we have proposed the linear matrix inequality based design method for multisynchronization 
control problem. From the previous discussion, linear matrix inequalities (13) and (14) in Theorem 5 and linear matrix inequalities (32) and (33) in Theorem 6 can be effectively solved.

Remark 8. When nonlinear systems generate multiple locally stable equilibria, finding appropriate control strategy and effective method to deal with such nonlinear systems is difficult. For example, as shown in [34], how to achieve the new synchronization scheme for multistable nonlinear systems is a very intractable problem. However, this issue may be solved if the effective impulsive control strategy is adopted.

Remark 9. Note that the impulsive control strategy (11) or (30) samples the state information only at impulsive times $t_{h}$; namely, each subnetwork takes only the sampling information of its neighbors. Hence, compared with the continuous control law, the impulsive control strategy then has strong pertinence, low energy consumption, and high response speed. As revealed in (12) and (31), the impulsive control system integrates the advantages of impulsive control and continuous control.

Remark 10. Under the framework of Filippov solution, $\mathrm{Gu}$ et al. [1] investigate the global synchronization of fractionalorder memristive neural networks based on comparison principle and Lyapunov method. Together with fractionalorder differential inequality and Lyapunov theory, Xiao et al. [8] analyze the finite-time synchronization of fractionalorder memristive bidirectional associative memory neural networks. By employing Holder inequality, $C_{p}$ inequality, and Gronwall-Bellman inequality, Yang et al. [9] formulate the quasi-uniform synchronization for fractional-order memristive neural networks. Based on Barbalat lemma and Razumikhin-type stability theorem, Zhang et al. [11] establish projective synchronization for fractional-order memristive neural networks. By using the infinitesimal generator on analytic semigroup principle and inequality techniques, Zhou et al. [12] study exponential synchronization of stochastic neural networks driven by fractional Brownian motion. By introducing the concept of joint connectivity and sequential connectivity, Chen et al. [21] show that complex networks can synchronize even if the topology is not connected at any time instant. By combining adaptive control and impulsive control, Yang et al. [17] discuss the global exponential synchronization of complex dynamical networks with nonidentical nodes and stochastic perturbation. By using the mathematical induction method, Zhang et al. [18] achieve the stochastic exponential synchronization for a class of delayed dynamical networks under delayed impulsive control, whereas the above works are all concerned about the global synchronization (or monosynchronization). These analytical methods for global synchronization (or monosynchronization) can not be migrated well to the multisynchronization problem. Using the impulsive control strategy and the Razumikhin-type technique, Wang et al. [16] study the multisynchronization problem of coupled neural networks with directed topology. Nevertheless, the controlled system in [16] is integer-order model. As have often been noted in most existing publications, analytical approach for integral-order systems could not be directly

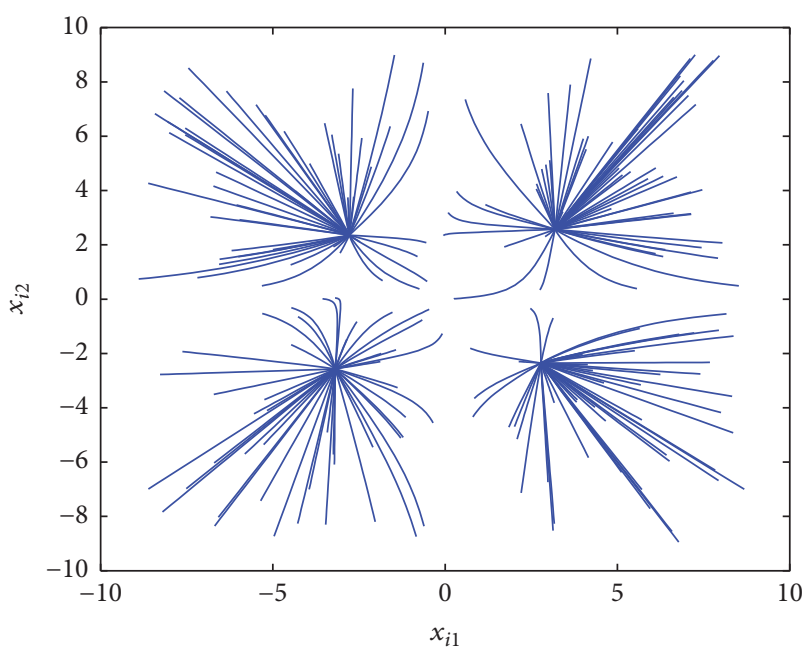

FIGURE 1: State evolutive characteristics.

extended and applied to deal with fractional-order systems. In this light, this paper extends and renews the relative results.

\section{Two Numerical Examples}

In the following numerical examples, we consider the coupled fractional-order neural networks consisting of three identical subnetworks; meanwhile, every subnetwork has two neuron states and the parameters of every subnetwork are characterized by

$$
\begin{aligned}
D & =\operatorname{diag}(1,1), \\
B & =\left[\begin{array}{cc}
3 & 0.2 \\
0.1 & 2.5
\end{array}\right], \\
f_{1}(s) & =f_{2}(s)=\tanh (s) .
\end{aligned}
$$

When there is zero input in every subnetwork above, conditions (2)-(6) are satisfied. That is, (A1)-(A3) hold. According to Lemma 1, every subnetwork has 4 locally Mittag-Leffler stable equilibria. Figure 1 depicts the state evolutive characteristics.

Example 11. We investigate fixed topology case. The Laplacian matrix $L=\left(\mathscr{L}_{i j}\right)_{3 \times 3}$ associated with digraph $\mathscr{D}$ is described as

$$
L=\left[\begin{array}{ccc}
1 & -1 & 0 \\
0 & 1 & -1 \\
-1 & 0 & 1
\end{array}\right]
$$

To choose constant $\alpha_{1}=0.3$, in addition, we select $\alpha_{2}=0.9, a=0.1$, and matrices $\mathscr{R}=\operatorname{diag}(1,1)$ and $\mathscr{W}=$ $\operatorname{diag}(0.5,0.5)$, and then conditions (13) and (14) are satisfied. That is, Theorem 5 holds. According to Theorem 5, the controlled system can achieve static multisynchronization. Figure 2 depicts the multisynchronization characteristics. 


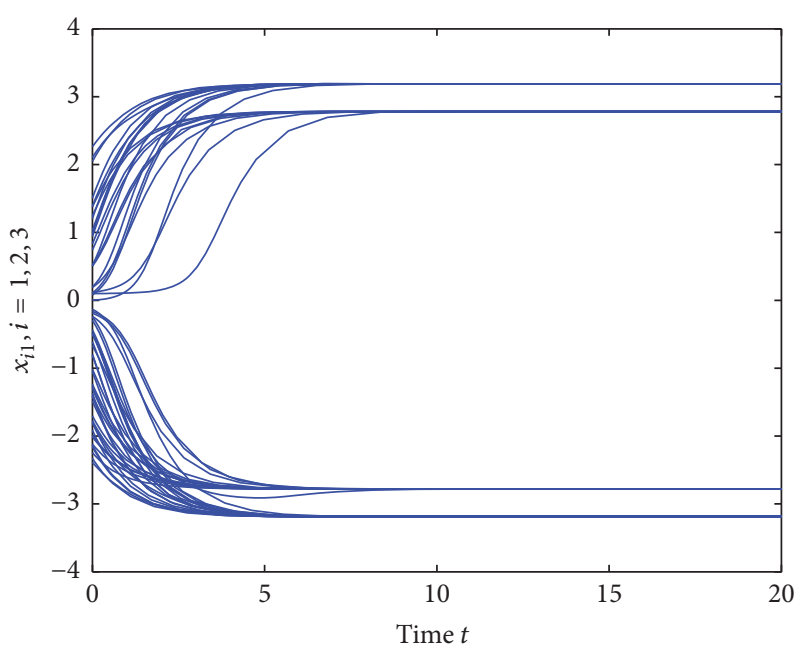

(a)

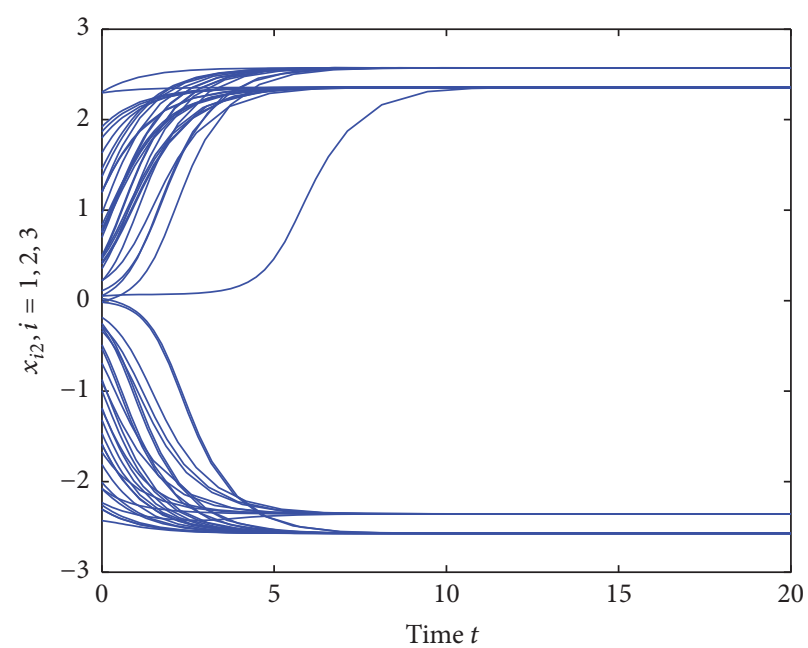

(b)

FIgURE 2: Multisynchronization characteristics in fixed topology case.

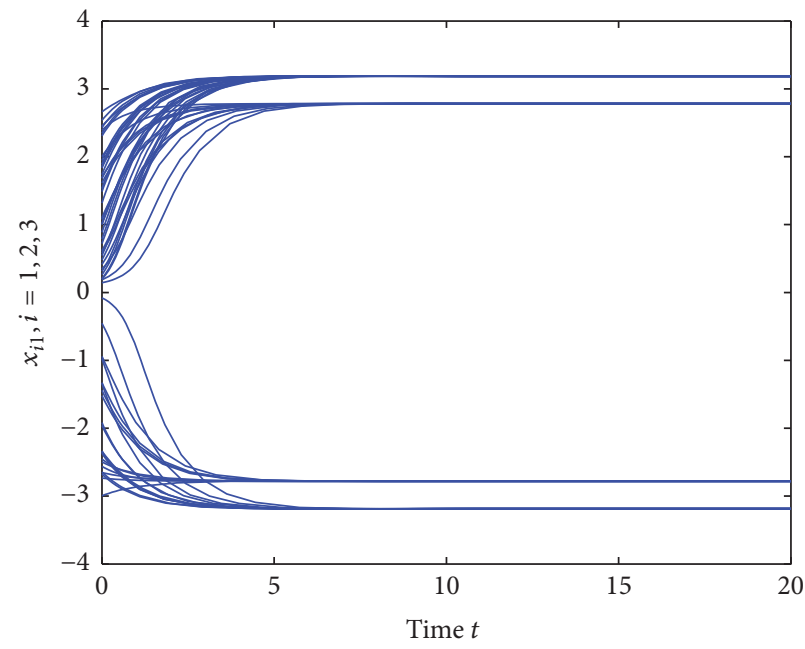

(a)

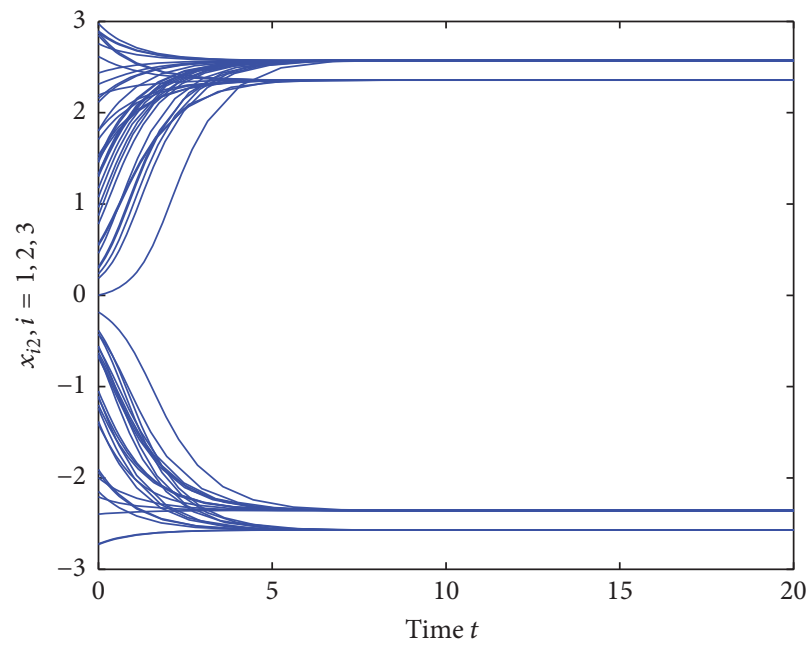

(b)

FIGURE 3: Multisynchronization characteristics in switching topology case.

Example 12. We investigate switching topology case. The Laplacian matrices $L^{\rho(t)}=\left(\mathscr{L}_{i j}^{\rho(t)}\right)_{3 \times 3}$ associated with digraphs $\mathscr{D}^{\rho(t)}$ are described as

$$
\begin{aligned}
L^{1} & =\left[\begin{array}{ccc}
1 & -1 & 0 \\
0 & 1 & -1 \\
-1 & 0 & 1
\end{array}\right], \\
L^{2} & =\left[\begin{array}{ccc}
1 & 0 & -1 \\
-1 & 1 & 0 \\
0 & -1 & 1
\end{array}\right],
\end{aligned}
$$

$$
L^{3}=\left[\begin{array}{ccc}
2 & -1 & -1 \\
-1 & 2 & -1 \\
-1 & -1 & 2
\end{array}\right]
$$

To choose constant $\alpha_{1}=0.3$, in addition, we select $\alpha_{2}=$ $0.9, a^{1}=0.1, a^{2}=0.1, a^{3}=0.3$, and matrices $\mathscr{R}=$ $\operatorname{diag}(1,1)$ and $\mathscr{W}=\operatorname{diag}(0.5,0.5)$, and then conditions (32) and (33) are satisfied. That is, Theorem 6 holds. According to Theorem 6, the controlled system can achieve static multisynchronization. Figure 3 depicts the multisynchronization characteristics. 


\section{Concluding Remarks}

Multisynchronization represents one of the most striking manifestations of multiconsistency in multistable systems. In this paper, we present the analytical results on the multisynchronization for coupled multistable fractional-order neural networks. Using the impulsive control principle, fractionalorder Lyapunov method, and linear matrix inequality technique, several sufficient conditions are deduced to guarantee multisynchronization which characterize the cardinality of the set of synchronization manifolds. How to develop new multisynchronization schemes for multistable fractionalorder systems would be the topic of future research.

\section{Conflicts of Interest}

The author declares that there are no conflicts of interest regarding the publication of this paper.

\section{Acknowledgments}

The work is supported by the Research Project of Hubei Provincial Department of Education of China under Grant T201412.

\section{References}

[1] Y. Gu, Y. Yu, and H. Wang, "Synchronization for fractionalorder time-delayed memristor-based neural networks with parameter uncertainty," Journal of the Franklin Institute. Engineering and Applied Mathematics, vol. 353, no. 15, pp. 36573684, 2016.

[2] P. Liu, Z. Zeng, and J. Wang, "Multiple Mittag-Leffler Stability of Fractional-Order Recurrent Neural Networks," IEEE Transactions on Systems, Man, and Cybernetics: Systems, vol. 47, no. 8, pp. 2279-2288, 2017.

[3] R. Rakkiyappan, J. Cao, and G. Velmurugan, "Existence and uniform stability analysis of fractional-order complex-valued neural networks with time delays," IEEE Transactions on Neural Networks and Learning Systems, vol. 26, no. 1, pp. 84-97, 2015.

[4] G. Velmurugan, R. Rakkiyappan, V. Vembarasan, J. Cao, and A. Alsaedi, "Dissipativity and stability analysis of fractionalorder complex-valued neural networks with time delay," Neural Networks, vol. 86, pp. 42-53, 2017.

[5] A. Wu and Z. Zeng, "Boundedness, Mittag-Leffler stability and asymptotical $\omega$-periodicity of fractional-order fuzzy neural networks," Neural Networks, vol. 74, pp. 73-84, 2016.

[6] A. Wu and Z. Zeng, "Global Mittag-Leffler stabilization of fractional-order memristive neural networks," IEEE Transactions on Neural Networks and Learning Systems, vol. 28, no. 1, pp. 206-217, 2017.

[7] A. Wu, L. Liu, T. Huang, and Z. Zeng, "Mittag-Leffler stability of fractional-order neural networks in the presence of generalized piecewise constant arguments," Neural Networks, vol. 85, pp. 118-127, 2017.

[8] J. Xiao, S. Zhong, Y. Li, and F. Xu, "Finite-time Mittag-Leffler synchronization of fractional-order memristive BAM neural networks with time delays," Neurocomputing, vol. 219, pp. 431439, 2017.
[9] X. Yang, C. Li, T. Huang, Q. Song, and X. Chen, "Quasiuniform synchronization of fractional-order memristor-based neural networks with delay," Neurocomputing, vol. 234, pp. 205215, 2017.

[10] L. Zhang, Q. Song, and Z. Zhao, "Stability analysis of fractionalorder complex-valued neural networks with both leakage and discrete delays," Applied Mathematics and Computation, vol. 298, pp. 296-309, 2017.

[11] L. Zhang, Y. Yang, and F. Wang, "Projective synchronization of fractional-order memristive neural networks with switching jumps mismatch," Physica A. Statistical Mechanics and its Applications, vol. 471, pp. 402-415, 2017.

[12] W. Zhou, X. Zhou, J. Yang, Y. Liu, X. Zhang, and X. Ding, "Exponential synchronization for stochastic neural networks driven by fractional Brownian motion," Journal of the Franklin Institute. Engineering and Applied Mathematics, vol. 353, no. 8, pp. 1689-1712, 2016.

[13] C. D. Cruz-Ancona, R. Martínez-Guerra, and C. A. PérezPinacho, "Generalized multi-synchronization: a leader-following consensus problem of multi-agent systems," Neurocomputing, vol. 233, pp. 52-60, 2017.

[14] R. Martinez-Guerra, C. D. Cruz-Ancona, and C. A. PerezPinacho, "Generalized multi-synchronization viewed as a multi-agent leader-following consensus problem," Applied Mathematics and Computation, vol. 282, pp. 226-236, 2016.

[15] H. Salarieh and M. Shahrokhi, "Multi-synchronization of chaos via linear output feedback strategy," Journal of Computational and Applied Mathematics, vol. 223, no. 2, pp. 842-852, 2009.

[16] Y.-W. Wang, W. Yang, J.-W. Xiao, and Z.-G. Zeng, "Impulsive multisynchronization of coupled multistable neural networks with time-varying delay," IEEE Transactions on Neural Networks and Learning Systems, vol. 28, no. 7, pp. 1560-1571, 2017.

[17] X. Yang, J. Cao, and J. Lu, "Stochastic synchronization of complex networks with nonidentical nodes via hybrid adaptive and impulsive control," IEEE Transactions on Circuits and Systems I: Regular Papers, vol. 59, no. 2, pp. 371-384, 2012.

[18] W. Zhang, Y. Tang, Q. Miao, and J.-A. Fang, "Synchronization of stochastic dynamical networks under impulsive control with time delays," IEEE Transactions on Neural Networks and Learning Systems, vol. 25, no. 10, pp. 1758-1768, 2014.

[19] M. Ayati and H. Khaloozadeh, "Designing a novel adaptive impulsive observer for nonlinear continuous systems using LMIs," IEEE Transactions on Circuits and Systems I: Regular Papers, vol. 59, no. 1, pp. 179-187, 2012.

[20] W.-H. Chen, D. Wei, and W. X. Zheng, "Delayed impulsive control of takagi-sugeno fuzzy delay systems," IEEE Transactions on Fuzzy Systems, vol. 21, no. 3, pp. 516-526, 2013.

[21] Y. Chen, W. Yu, F. Li, and S. Feng, "Synchronization of complex networks with impulsive control and disconnected topology," IEEE Transactions on Circuits and Systems II: Express Briefs, vol. 60, no. 5, pp. 292-296, 2013.

[22] M. Claeys, D. Arzelier, D. Henrion, and J.-B. Lasserre, "Measures and LMIs for impulsive nonlinear optimal control," IEEE Transactions on Automatic ControL, vol. 59, no. 5, pp. 1374-1379, 2014.

[23] L. Ding, P. Yu, Z.-W. Liu, and Z.-H. Guan, "Consensus and performance optimisation of multi-agent systems with positiononly information via impulsive control," IET Control Theory \& Applications, vol. 7, no. 1, pp. 16-24, 2013.

[24] W. Du, S. Y. S. Leung, Y. Tang, and A. V. Vasilakos, "Differential evolution with event-triggered impulsive control," IEEE Transactions on Cybernetics, vol. 47, no. 1, pp. 244-257, 2017. 
[25] S. L. Fraga and F. L. Pereira, "Hamilton-Jacobi-Bellman equation and feedback synthesis for impulsive control," IEEE Transactions on Automatic Control, vol. 57, no. 1, pp. 244-249, 2012.

[26] D. He and L. Xu, "Ultimate Boundedness of Nonautonomous Dynamical Complex Networks under Impulsive Control," IEEE Transactions on Circuits and Systems II: Express Briefs, vol. 62, no. 10, pp. 997-1001, 2015.

[27] X. Li and S. Song, "Stabilization of Delay Systems: delaydependent Impulsive Control," IEEE Transactions on Automatic Control, vol. 62, no. 1, pp. 406-411, 2017.

[28] Y. Li, Y. Sun, J. Hua, and L. Li, "Indirect adaptive type-2 fuzzy impulsive control of nonlinear systems," IEEE Transactions on Fuzzy Systems, vol. 23, no. 4, pp. 1084-1099, 2015.

[29] X. Liu and K. Zhang, "Impulsive control for stabilisation of discrete delay systems and synchronisation of discrete delay dynamical networks," IET Control Theory \& Applications, vol. 8, no. 13, pp. 1185-1195, 2014.

[30] Y. Liu, S. Zhao, and J. Lu, "A new fuzzy impulsive control of chaotic systems based on T-S fuzzy model," IEEE Transactions on Fuzzy Systems, vol. 19, no. 2, pp. 393-398, 2011.

[31] X. Lu, N. Chen, Y. Wang, L. Qu, and J. Lai, "Distributed impulsive control for islanded microgrids with variable communication delays," IET Control Theory \& Applications, vol. 10, no. 14, pp. 1732-1739, 2016.

[32] T. H. Cormen, C. E. Leiserson, R. Rivest, and C. Stein, Introduction to Algorithms, The MIT Press, Cambridge, Massachusetts, Mass, USA, 2009.

[33] S. Boyd, L. El Ghaoui, E. Feron, and V. Balakrishnan, Linear Matrix Inequalities in System and Control Theory, SIAM, Philadelphia, PA, USA, 1994.

[34] A. Wu and Z. Zeng, "Output convergence of fuzzy neurodynamic system with piecewise constant argument of generalized type and time-varying input," IEEE Transactions on Systems, Man, and Cybernetics: Systems, vol. 46, no. 12, pp. 1689-1702, 2016. 


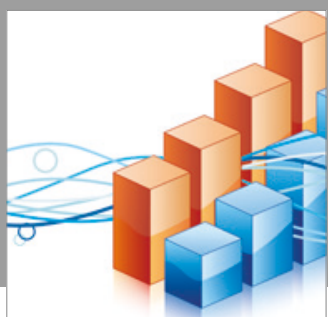

Advances in

Operations Research

vatersals

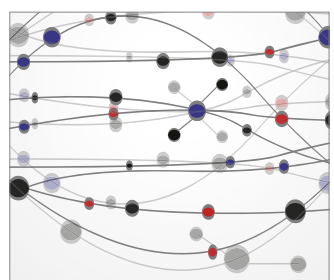

\section{The Scientific} World Journal
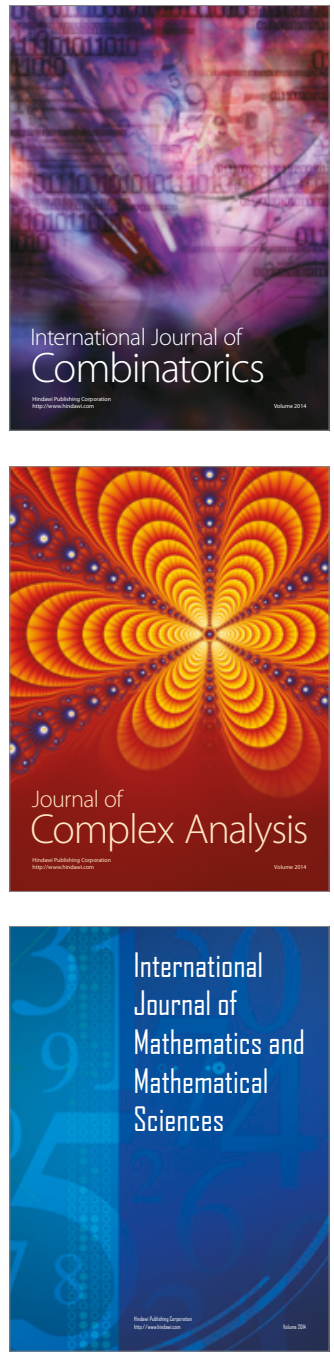
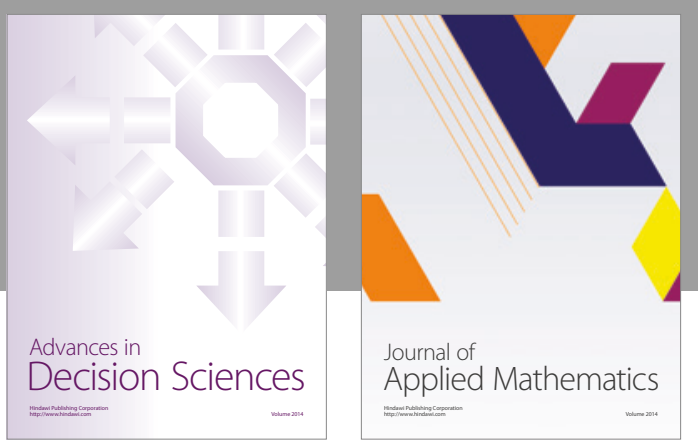

Algebra

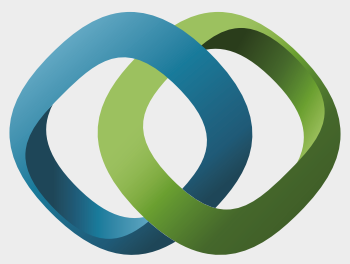

\section{Hindawi}

Submit your manuscripts at

https://www.hindawi.com
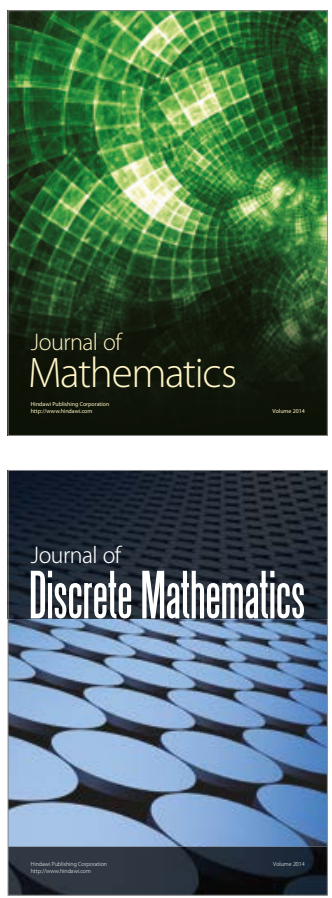

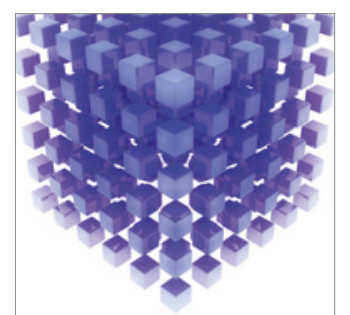

Mathematical Problems in Engineering
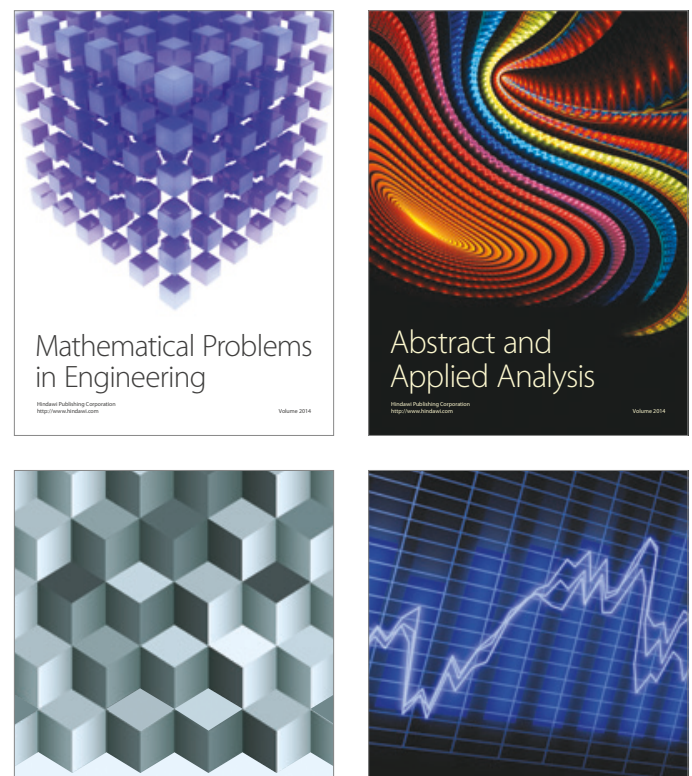

Journal of

Function Spaces

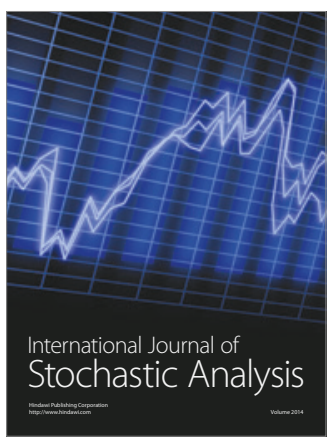

Probability and Statistics
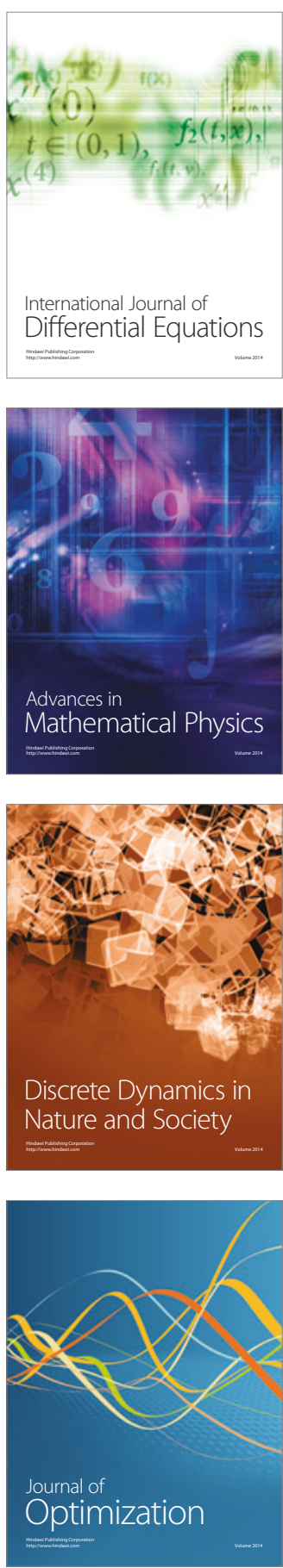